\title{
Magnetic Assisted Laser Micromachining for Highly Reflective Metals
}

\author{
Yuan-Jen Chang, Chia-Lung Kuo, and Nai-Yu Wang \\ Department of Mechanical Engineering, National Yunlin University of Science and Technology, \\ No. 123, Sec. University Rd., Douliou, Yunlin 640 Taiwan, R.O. C. \\ E-mail: changy@yuntech.edu.tw
}

\begin{abstract}
In this paper, we report the results of laser machining on highly reflective materials, such as Al6061, with an assisting magnetic field. Both static and dynamic magnetic fields are set so as to examine their effect on both the laser machining efficiency and the inlet diameter. During the laser machining, the Lorentz force generated by the assisting magnetic field affects the laser-induced plasma and results in enhanced performance of the laser machining. With a proper arrangement of the static magnetic field, the machining depth can be increased by $266 \%$, and the inlet diameter can be reduced by $42 \%$. With a rotational dynamic magnetic field, both the roundness of the inlet and the HAZ can be improved. DOI:10.2961/jlmn.2012.03.0004
\end{abstract}

Keywords: Laser, Magnetic field, Reflective Surface, Micromachining, Laser drilling.

\section{Introduction}

Laser beam processing has advanced significantly over the past few decades. It provides high machining accuracy and high machining speed for a wide range of materials. Practically, $\mathrm{CO}_{2}$ and $\mathrm{Nd}$ :YAG lasers are commonly employed as the laser source, due to their excellent performance and affordability. However, laser precision machining encounters difficulty in applications with a highly reflective target surface, which reflects the optical energy and dramatically reduces the processing efficiency. It lengthens the cutting time, increasing the cost of the process and decreasing the yield. Several solutions have been proposed to overcome this issue. Thawari et al. use high power lasers to provide enough energy to penetrate the highly reflective surface without reducing the yield of the process, but efficiency remains low [1]. Gu et al. report that shorter wavelength lasers improve absorption by the reflective material, thereby providing a higher cutting speed [2]. Zhu et al. utilize an ultra-fast pulsed laser, such as a femtosecond laser, to provide high cutting speed without the effect of reflectivity from surfaces [3]. Both methods require expensive equipment, which increases the cost of machining.

A magnetic field has been used in previous research to assist the laser welding by a $\mathrm{CO}_{2}$ laser in order to provide better machining performance [4][5]. An assisting magnetic field can also be employed to guide the laser-produced plasma in pulsed laser deposition [6][7]. The dynamics of the laser-induced plasma in laser machining are also investigated [8][9]. The studies show that the laser induced plume in the machining process can be confined with an assisting magnetic field in order to increase its plasma density. To the best of our knowledge, the effect of an assisting magnetic field on laser drilling has not been investigated before. In this paper, we report the effect of both of static and dynamic magnetic fields on laser drilling. The hole profiles and laser machining efficiency are examined and measured. The results show that the machining depth can be increased by $266 \%$ and the inlet diameter reduced by $42 \%$, with the proper arrangement of the static magnetic field. With a rotational dynamic magnetic field, the roundness of the inlet and the HAZ can be improved.

\section{Experimental Setup}

In our experiments, two different magnetic fields: static and dynamic, generated by permanent magnets were used. The whole setup was established on a Sodick AP1L Micro Precision EDM machine. A fixture connected the holders of the optical components, a mirror and a lens, to the column of the EDM machine. The mirror reflected the laser to the sample while the lens with a Z-axis-stage focused the laser onto the sample. The sample, an Al6061 plate, was secured on a precision vice affixed on the XY stage of the EDM machine. The laser source was a Lotis 213 UTF Nd:YAG Laser which provided four harmonic modes, i.e. four different wavelengths. The $532 \mathrm{~nm}$ wavelength with pulse mode was used in all of our experiments. The maximum energy of a single pulse was $270 \mathrm{~mJ}$ at this wavelength, while the maximum frequency was $15 \mathrm{~Hz}$. The pulse width was around 6ns. For Nd:YAG lasers, Al6061 has a high reflectivity (over 90\%). Therefore, the machining efficiency for this material is low. Meanwhile, the specific heat of Al6061 $\left(0.9 \mathrm{~J} / \mathrm{g}^{\circ} \mathrm{C}\right)$ is higher than other metals like $\mathrm{Au}$ $\left(0.129 \mathrm{~J} / \mathrm{g}^{\circ} \mathrm{C}\right)$, and $\mathrm{Cu}\left(0.385 \mathrm{~J} / \mathrm{g}^{\circ} \mathrm{C}\right)$. Thus, the machining efficiency deteriorated.

For a static magnetic field, we began the experiments with a simple setup as shown in Figure 1. The NdFeB permanent magnets were directly attached to the sample. The same number of magnets was placed on both sides of the sample to provide a symmetrical static magnetic field. We also positioned the multiple pairs of assembled magnets in a vertical arrangement to study their effects on the laser machining. The details will be discussed in the next section. The sample consisted of two pieces of Al6061 plate, and 
the drilling spot was at the interface of the two plates. The number of laser pulses was 225 for each experiment.

In order to generate a dynamic magnetic field, the experimental setup was modified, as shown in Figure 2. A set of assembled permanent magnets on a ball bearing, resulting in the driven set, produced a dynamic magnetic field. The permanent magnets were $5 \mathrm{~mm}$ in diameter and each assembled magnet consisted of two magnets. The assembled magnets were evenly distributed on the circumference of the ball bearing. The driven set was then fitted into one end of a hollow brass tube, which provided a pathway to the laser beam. The driven set was affixed to another precision vice. The distance from the bottom of the bearing to the sample was kept at $2 \mathrm{~mm}$. Arrangements of both one and three pairs of assembled magnets were investigated. The driving set had a pair of two assembled permanent magnets and a motor, as shown in Figure 2. The diameter of the magnets on the driving set was $10 \mathrm{~mm}$. The larger magnets provided a wider range of magnetic force, ensuring smooth rotation of the driven set. The distance from the driving set to the driven set was $15 \mathrm{~mm}$. The rotational speed of the driven set followed the rotational speed of the motor on the driving set.

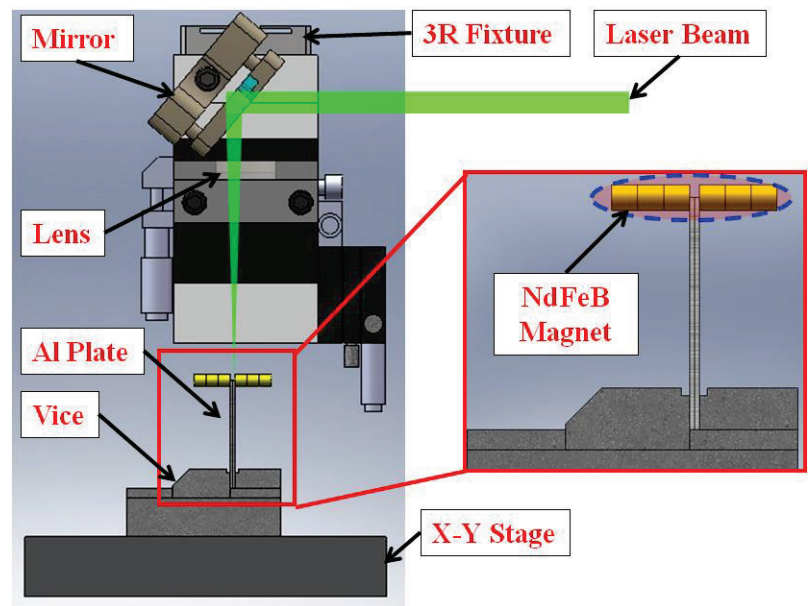

Figure 1 Schematic of experimental setup with a static magnetic field by attaching permanent magnets directly to the sample

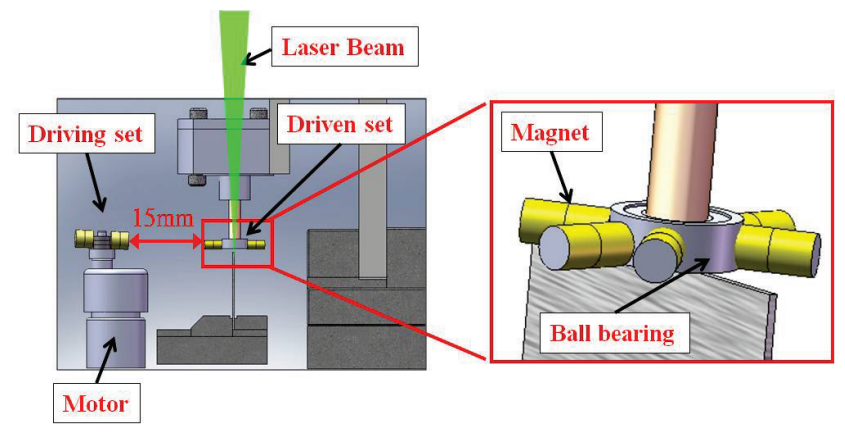

Figure 2 Schematic of experimental setup with a dynamic magnetic field

\section{Results and Discussion}

First, we proved that the static magnetic fields do affect the laser-induced plume, by observing the change of the height under different strengths of magnetic field. Second, two different arrangements were performed to investigate the effect of different static magnetic fields on laser drilling. Finally, dynamic magnetic fields were applied to investigate their effect on laser drilling. The profiles, depth and inlet diameter of the machined holes were all recorded and the reasons were discussed for each case.

\subsection{Height of Laser-Produced Plasma}

We investigated the height of laser-produced plasma with different strengths of static magnetic fields. The investigation was performed with the setup shown in Figure 1. Three different positions of magnets were employed in the measurements. A CCD recorded the images of the plasma with a pair of goggles in front of the CCD for protection. The number of magnets was varied from one to four on both sides of the sample. First, the tops of the magnets were positioned $2 \mathrm{~mm}$ above the top of the sample. The strength of the magnetic field at the middle of the top surface of the sample was measured by a Gauss-meter. It increased with the number of the magnets, ranging from 1666 Gauss to 4230 Gauss. The height of the laser-produced plasma increased with the number of magnets as well. Two more arrangements were tested; one at the same height as the sample and the other, $2 \mathrm{~mm}$ below the top surface of the sample. The results are shown in Figure 3. The strengths in the second arrangement ranged from 189 Gauss to 530 Gauss, while those in the third arrangement ranged from 154 Gauss to 79 Gauss. With the smaller strength in the last two arrangements, the plasma was shorter. Moreover, these arrangements with four magnets showed a much lower magnetic field strength, and resulted in even shorter plasma.

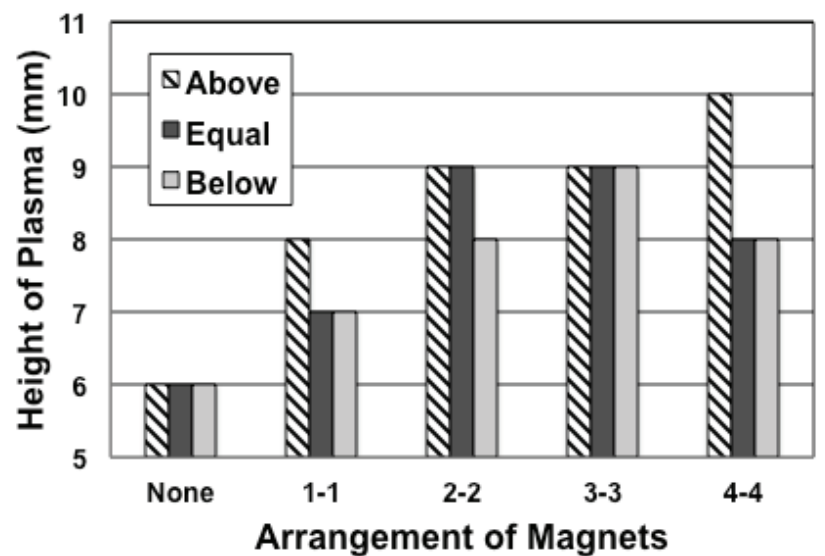

Figure 3 Height of plasma with different arrangements of magnets

\subsection{Static Magnetic Field}

Two different arrangements for generating a static magnetic field were examined in our experiments. One was a single pair of assembled magnets on both sides of the sample, and the other consisted of multiple pairs of assembled magnets in a vertical arrangement.

\subsubsection{Single pair of magnets}

The pairs of one to four magnets were tested in this arrangement. Three different positions, as described above, 
of the pairs were examined. From the profiles of the drilling holes shown in Figure 4, the depth with the pairs of the magnets was deeper than without the magnetic field. The deepest hole occurred with the pair of single magnets. The curves clearly shown inside the holes by the pairs of single or two magnets are believed to be the recasting of removed material on the hole sidewall. The melted material could not be removed from the hole due to its depth. With the pairs of three and four magnets, the profiles show the amphoral cross-section. We believe it was due to the bombardment of laser-induced plasma by the Lorentz force from the strong magnetic field. The profiles showed that the stronger a magnetic field, the more bombardment of the plasma in the hole.

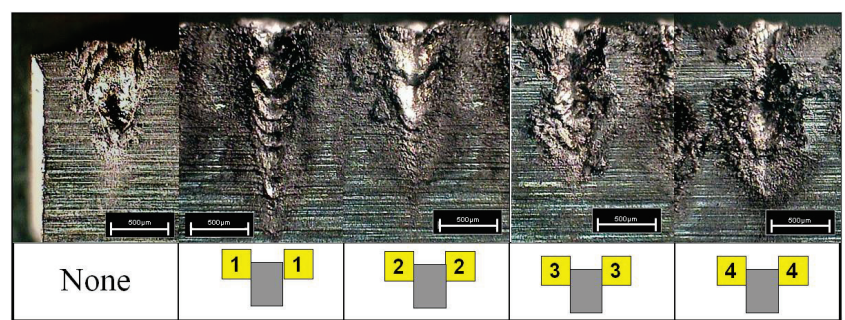

Figure 4 Profiles of drilling holes with different numbers of magnets (scale bars shown are $500 \mu \mathrm{m}$ )

The measured depths and inlet diameters of the holes are shown in Figure 5. The depth of the hole with a pair of single magnets reached around $1400 \mu \mathrm{m}$, a $133 \%$ increase to the hole without a magnetic field. The inlet diameter was reduced by $33 \%$.

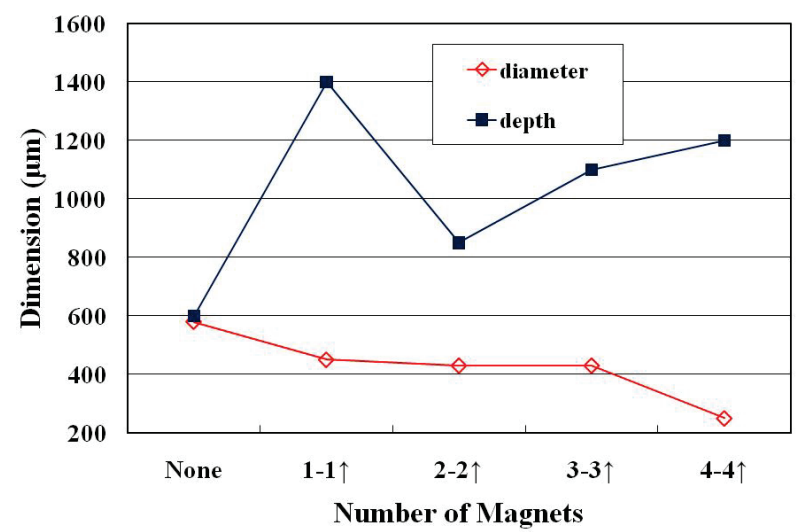

Figure 5 Depth and inlet diameter of holes with magnets $2 \mathrm{~mm}$ above the sample

For the pairs of magnets placed at the same height as the top of the sample, the profiles clearly showed the confinement of the laser-induced plasma from the shrinkage of the inlet diameter. The recasting material was still seen in the holes with the pairs of three and four magnets. The depths were not as deep as those in the previous case. Moreover, the depths were less than that of the hole without a magnetic field. The enhanced bombardment was also observed with the pair of four magnets.

The measured depths and inlet diameters of the holes are shown in Figure 7. Although the inlet diameters were reduced in all cases, the depths were reduced by $17 \%$ and $33 \%$ with the pairs of one and two magnets, respectively.

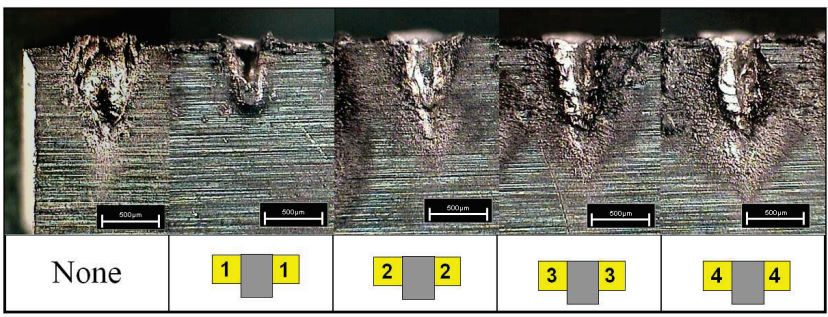

Figure 6 Profiles of holes with magnets at the same height of sample top surface (scale bars shown are $500 \mu \mathrm{m}$ )

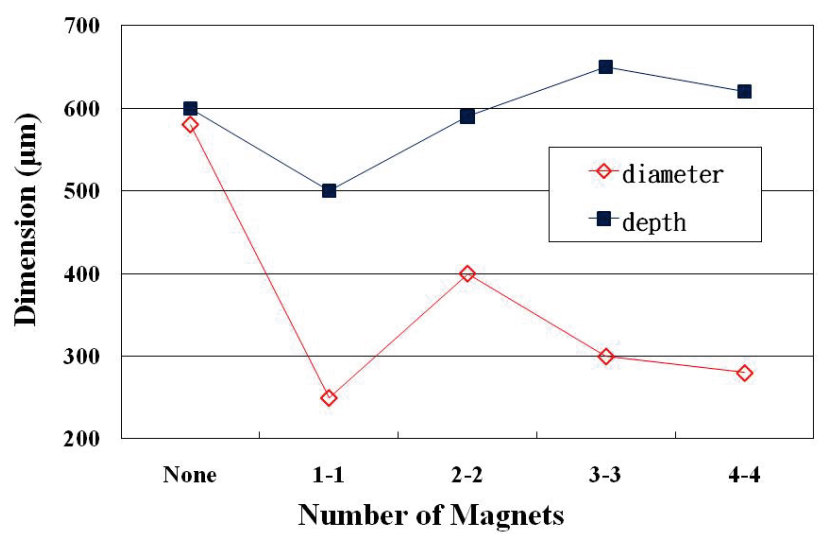

Figure 7 Depth and inlet diameter of drilling hole with magnets at the same height as sample top surface

We then placed the pairs of magnets $2 \mathrm{~mm}$ below the top of the sample to examine the drilling performance. The profiles of the drilling holes are shown in Figure 8. They show that the narrow-neck shape appeared at the inlets. The measured depths and inlet diameters are shown in Figure 9. The depths of the holes were all deeper than those in the previous case. Moreover, the depths were all greater than that of the hole without a magnetic field. The deepest hole occurred with the pair of three magnets. With the pair of four magnets, the magnetic strength at the spot of machining was measured to be less than with three magnets, resulting in a shallower hole.

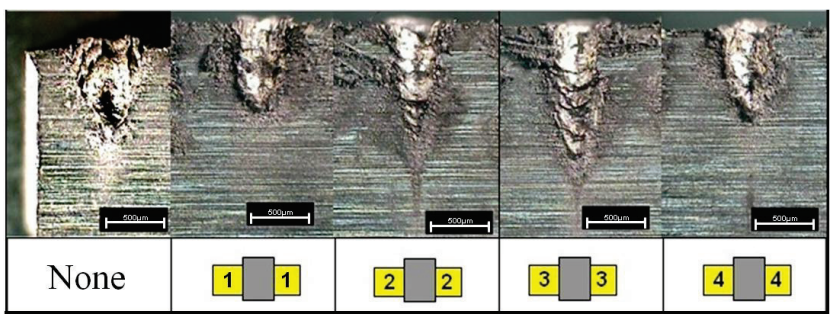

Figure 8 Profiles of drilling holes with magnets $2 \mathrm{~mm}$ below sample (scale bars shown are $500 \mu \mathrm{m}$ )

\subsubsection{Multiple pairs of magnets}

We also investigated the effect of multiple pairs of assembled magnets in a vertical arrangement on the laser drilling. Four arrangements, shown in Figure 10, were tested. 


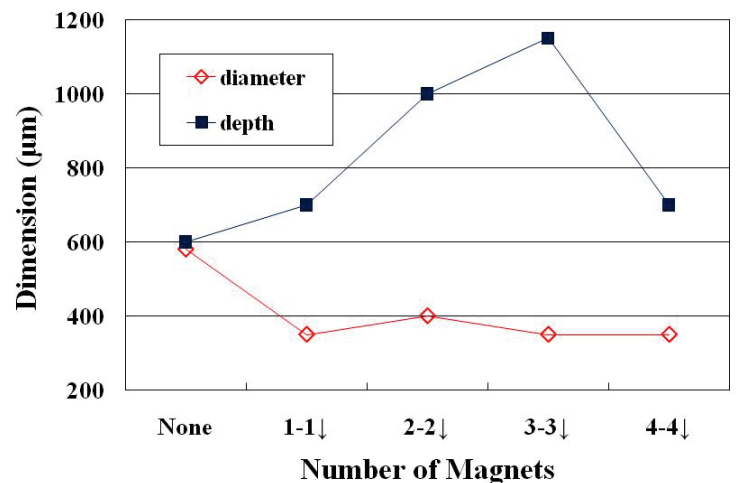

Figure 9 Depth and inlet diameter of holes with magnets $2 \mathrm{~mm}$ below sample
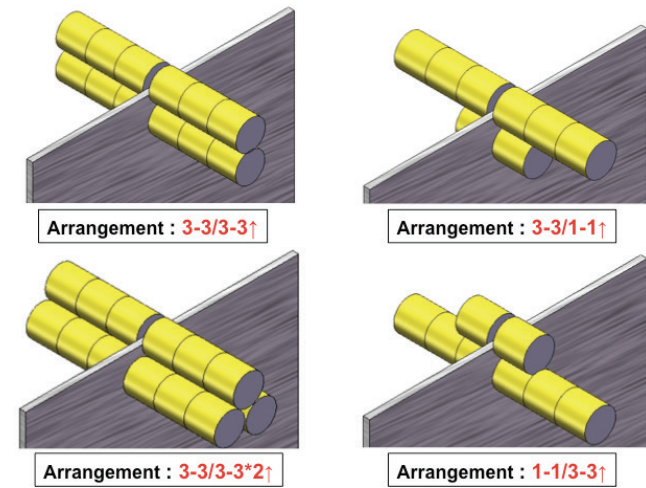

Figure 10 Vertical arrangements of multiple magnetic pairs

First, the top pair was placed $2 \mathrm{~mm}$ above the surface of the sample. The profiles, measured hole depths, and inlet diameters are shown in Figure 11 and Figure 12. The pro-

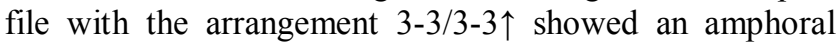
shape, indicating strong plasma confinement at the inlet and bombardment inside the hole. We believed that the lower pair of assembled magnets enhanced the magnetic field and resulted in the strong bombardment. The depth with arrangement $3-3 / 3-3 * 2 \uparrow$ reached $1600 \mu \mathrm{m}$ and there was no bombardment visible inside the hole. We believe that this was because the positions of the two pairs of assembled magnets shifted from the machining spot and provided a downward bombardment inside the hole. The depth with arrangement 1-1/3-3个 showed a depth less than those of the other arrangements. We believe that this was due to the less efficient machining at the inlet with a pair of single magnets.

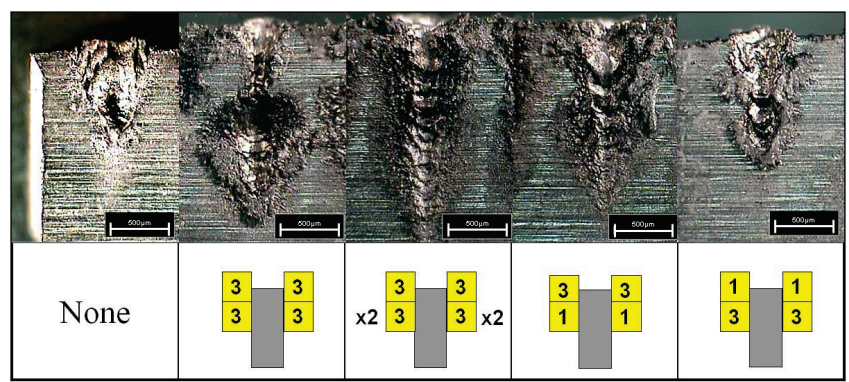

Figure 11 Profiles of holes with vertical arrangements of multiple assembled magnets $2 \mathrm{~mm}$ above sample (scale bars shown are $500 \mu \mathrm{m})$

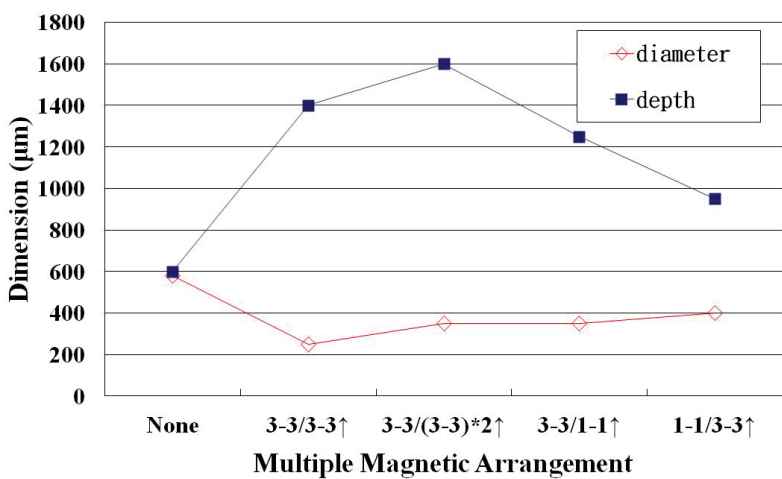

Figure 12 Depth and inlet diameter of drilling holes with multiple assembled magnets $2 \mathrm{~mm}$ above sample (scale bars shown are $500 \mu \mathrm{m})$

We then placed the vertical arrangements at the same height as the sample surface in order to compare the results with the previous cases. The profiles and measured depths and inlet diameters are shown in Figure 13 and Figure 14. The comparison between this arrangement and the previous case is also shown in Figure 14. With arrangement 3-3/3-3, it can clearly be seen that the profile did not show the amphoral shape, and the depth was less than arrangement 3$3 / 3-3 \uparrow$. The profile, depth and inlet diameter with arrangement $3-3 / 3-3 * 2$ were similar to those with arrangement 3 $3 / 3-3 * 2 \uparrow$. The depth with arrangement 3-3/1-1 reached $1640 \mu \mathrm{m}$, while the inlet diameter was reduced to $280 \mu \mathrm{m}$. The magnetic field provided a better confinement of the plasma and formed a downward Lorentz force to increase the machining efficiency.

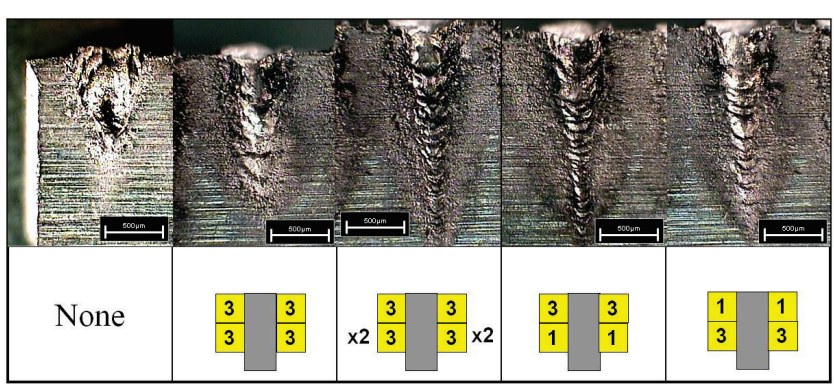

Figure 13 Profiles of holes with vertical arrangements of multiple assembled magnets at same height of sample (scale bars shown are $500 \mu \mathrm{m})$

From the above experimental results, the amphoral holes were obtained by laser machining with some arrangements of magnets. With an external magnetic field, a laser-induced plasma is expanded [10][11] or confined[12][13] depending on the orientation of the magnetic field. In our case, the laser-induced plasma is perpendicular to the magnetic field and expands in the direction along the magnetic field if the strength of the field is large enough [8]. Meanwhile, the ion density is higher on the outer shell of the plasma than it inside the plasma due to the Lorentz force. We believe that the expanding of laser-induced plasma increases the removal rate of material on the sidewalls and results in the amphoral shape. 


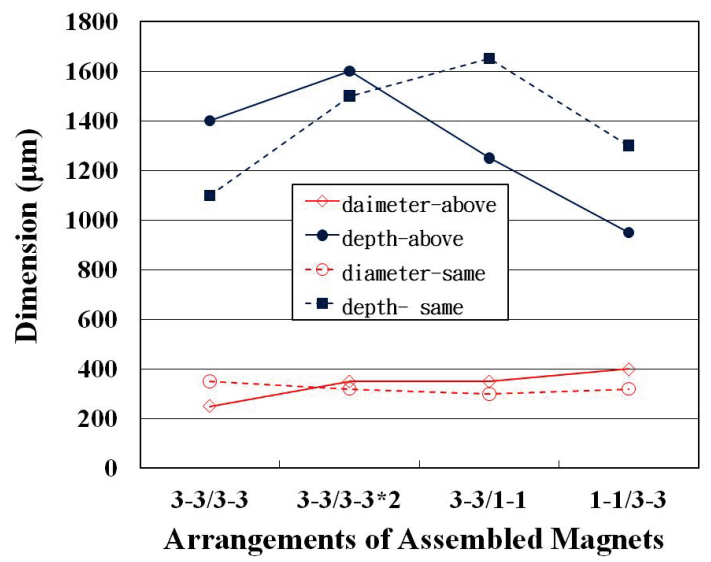

Figure 14 Comparison of depths and inlet diameters of holes with multiple assembled magnets at different positions

\subsection{Dynamic Magnetic Field}

The experiments with a dynamic magnetic field were performed with the second setup described in the Experimental Setup Section. Three different spinning speeds: 0rpm, 100rpm and 350rpm, of the driving set were used to present the different dynamic performance of a magnetic field. We also investigated the machining performance with two different arrangements of magnets, one pair and three pairs of the assembled magnet set. Each assembled magnet had two permanent magnets. The pictures of resulting inlets are shown in Table 1 and Table 2. The measured inlet diameters and depths of holes are shown in Figure 15. Compared to the hole with no magnetic field, the inlet diameter with a stationary pair of assembled magnets was reduced by about $30 \%$, and the heat affected zone (HAZ) was reduced by $37 \%$. The diameter and depth, however, were not effectively improved with the increased spinning speed. We believe it was due to the non-uniform distributed magnetic field between the driving and driven sets resulting in an unstable rotation of the single pair of assembled magnets.

Table 1 Pictures of inlets with one pair of assembled magnet set at different spinning speeds (scale bars shown are $500 \mu \mathrm{m})$

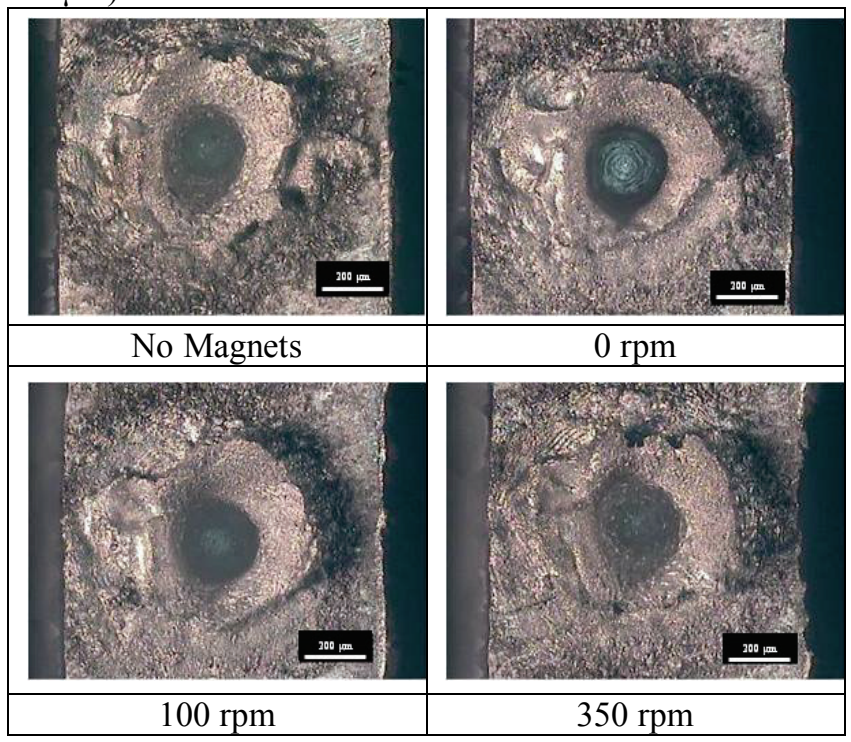

\section{Conclusions}

The effect of a magnetic field, both static and dynamic, on the performance of laser drilling was studied and reported in this paper. The Lorentz force was the main force confining the laser-induced plasma at the inlet and assisting the laser machining inside the hole.

For the static magnetic field generated by the single pairs, the machining efficiency was better when the magnets were positioned $2 \mathrm{~mm}$ above the sample. With one pair of single magnets placed $2 \mathrm{~mm}$ above the sample surface, the machining efficiency was highest, while the inlet diameter was the smallest if they were placed $2 \mathrm{~mm}$ below the sample surface.

For the static magnetic field generated by multiple pairs of the assembled magnets, arrangement $3-3 / 3-3 * 2 \uparrow$ provided the best result to both the machining efficiency and the inlet diameter.

For the dynamic magnetic field generated by rotational assembled magnets, a smooth magnetic field improved the roundness of the inlet, and the HAZ was reduced.

Table 2 Pictures of inlets with three pairs of assembled magnets set at different spinning speeds (scale bars shown are $500 \mu \mathrm{m})$
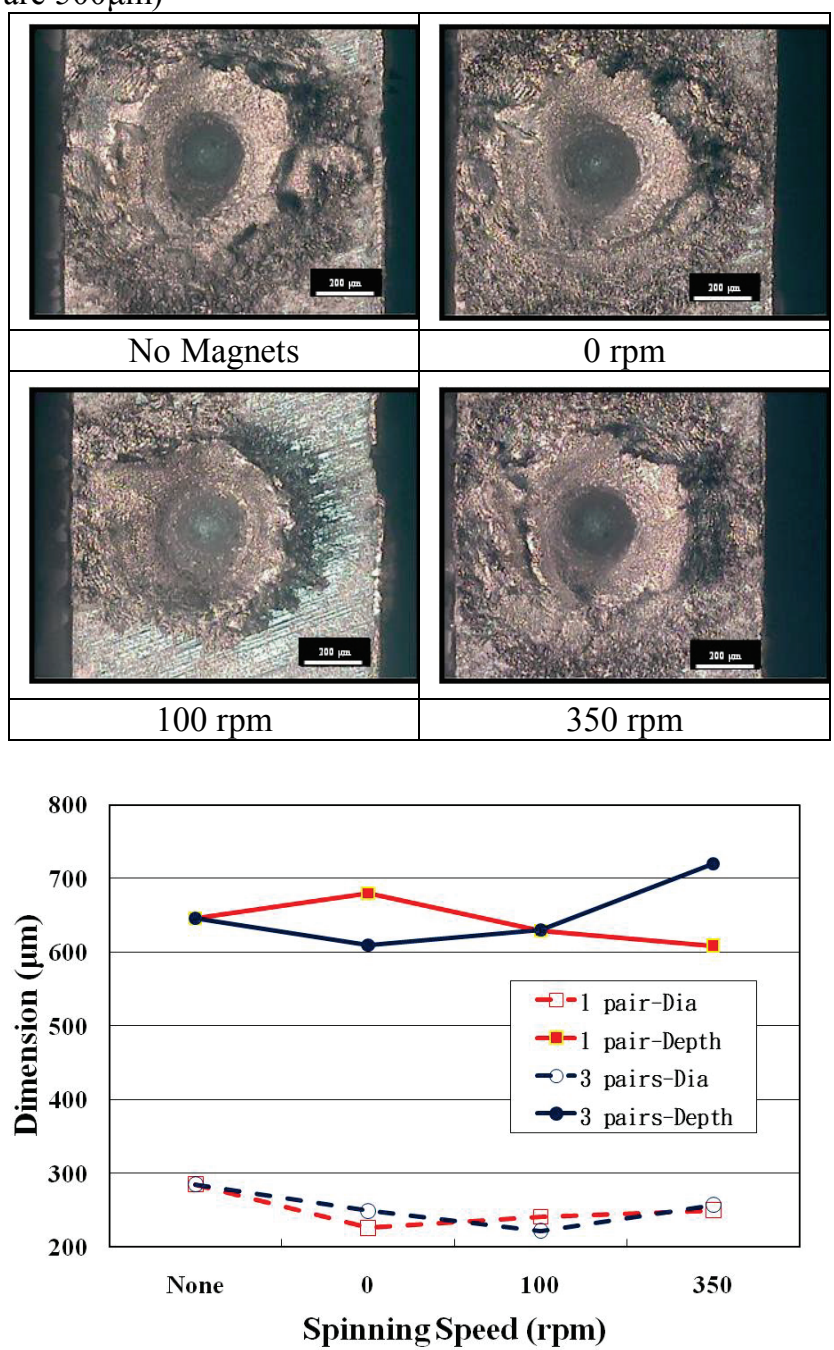

Figure 15 Inlet diameter and depth with different pairs of assembled magnets at different spinning speeds 


\section{Acknowledgments}

The authors, Kuo, C. L. and Wang, N. Y., gratefully acknowledge the financial support of the National Science Council of Taiwan, R.O.C. (NSC 97-2221-E-224-008MY3).

\section{References}

[1] Thawari, G., Sundar, J. K. Sarin, Sundararajan, G., and Joshi, S. V., J. of Mater. Process. Tech., 170, (2005), 299-239.

[2] Gu, E., Jeona, C. W., Choia, H. W., Ricea, G., Dawson, M. D., Illy, E. K., and Knowlesb, M. R. H., Thin Solid Films, 453 -454, (2004), 462-466

[3] Zhu, X., Villeneuve, D. M., Naumov, A. Yu., Nikumb, S., and Corkum, P. B., Appl. Surf. Sci., 152, (1999), 138-148.

[4] Tse, H.C., Man H. C. and Yue, T. M., Opt. and Laser in Engineering, 32, (1999), 55-63.
[5] Zhou, J, and Tsai, H.-L., Int'l J. of Heat and Mass Transfer, 50, (2007), 2217-2235.

[6] Tsui, Y.Y., Minami, H., Vick, D., and Fedosejevs, R., J. Vac. Sci. Technol. A, 20, (2002), 744-747.

[7] Garcia, T., de Posada, E, and Villagran, M., Appl. Surf. Sci., 255, (2008), 2200-2204.

[8] Harilal, S.S., Bindhu, C. V., and Tillack, M. S., Phys. Rev. E, 69, (2004), 026413.

[9] Qindeel, R., Bidin, N. B., and Mat daud, Y. J. of Phys.: Conf. Series, 142, (2008), 012069.

[10]Pramod, K. and Thareja, R. K., J. of Appl. Phys., 109, (2011), 074901.

[11]Pagano, C., Hafeez, S., and Lunney, J. G., J. Phys. D: Appl. Phys., 42, (2009), 155205.

[12]Mostovych, A. N., Ripin, B. H., and Stamper, J. A., Phys. Rev. Lett., 62, (1989), 2837-2840.

[13]Pagano, C., and Lunney, J. G., J. Phys. D: Appl. Phys., 43, (2010), 305202.

(Received: June 07, 2011, Accepted: June 13, 2012) 